\title{
Fission fragments of actinide and superheavy nuclides in primordial material of the Solar system and the problem of their origin
}

\author{
Georgy N. Goncharov \\ Department of Geochemisrty, St.-Petersburg State University \\ Universitetskaya nab. 7/9, 190034 St.-Petersburg, Russia. \\ E-mail: ggontcharov@mail.ru
}

Results of an estimation of the possible contribution of fission products into the abundance of atomic masses for transiron elements and xenon isotopes in different objects of the Solar system are presented in the work. A recent systematization of the mass distribution of fission fragments for actinide nuclides and compound nuclei of superheavy elements at low excitation energies has allowed to reveal the humps on the mass distribution curves of Solar system matter that are analogous to the mass fragment distributions at asymmetric fission for nuclei of thorium and uranium, and superheavy elements $(Z \approx 114$ and 126). These results are in accordance with the ratios of heavy xenon isotopes, found out in products of fragmental fission. Two basic sources of fission fragments in transiron area are revealed for matter of the Solar system. The first of them, connected with the fission of superheavy progenitors and characterized by Dy, Pt and Te enrichment ( $A=164,195$ and 130), is typical for carbonaceous chondrites and the Solar photosphere. The second one, connected with Th and $U$ fission and having the maxima of $\mathrm{Sr}, \mathrm{Zr}$ and $\mathrm{Ba}$ abundances $(\mathrm{A}=88,94$ and 138) is observed in the crusts of the Earth and the Moon. In the suggested scenario the basic phase of $r$ - process occurred after a synthesis of actinide and superheavy nuclides and was the reason of local modifications in the humps of their fission fragments distributions which coincide with the "waiting points" of $r$ - process.

International Symposium on Nuclear Astrophysics - Nuclei in the Cosmos - IX

CERN, Geneva, Switzerland

25-30 June, 2006 


\section{Introduction}

Abundances of chemical elements in different objects of the Solar system are kept substantially constant and equal to the ratios established during star nucleosynthesis. Moreover many of these ratios were formed in internal shells of stars which cannot be subjected to a spectroscopic study now. The information on it is "stored" in elemental abundances in nondifferentiated objects or in an average composition of the big bodies in the case of local migration of matter in cosmochemical and geochemical processes. It is possible to use these abundances for search of signatures characteristic for distribution of fragmental fission of the heaviest nuclei formed in star nucleosynthesis on the base of construction of atomic masses distributions for transiron elements in objects of the Solar system. $r$ - Process scenarios with fission recycling do not allow so far to determine parental fissioning nuclides.

It has been shown earlier [1], that the humps on a curve of atomic mass distribution in carbonaceous chondrites $(\mathrm{A}=130,164$ and 195) can be approximated by distributions of light and heavy fragments after they emit neutrons and undergo $\beta$ - decay. These fragments could be products of asymmetric fission of the progenitors having $Z=114_{-1}^{0}$ and $Z=126_{-2}^{0}$. The received result appears to be well coordinated with a systematization of mass distribution of fission fragments of compound nuclei in the superheavy region [8].

Actinide elements have a low abundance in carbonaceous chondrites and their fission fragments of primordial nucleosynthesis are poorly visible in a mass distribution curve for these meteorites. Objects which are enriched in uranium and thorium with the enrichment of at least two or three orders higher than in carbonaceous chondrites, are of special interest in this case. However, such objects are connected to the differentiated bodies and only their average composition can approach the element distribution in star nucleosynthesis. Fe nuclei might be seen as seeds for nucleogenesis of the heaviest elements in various scenarios. Normalizing of abundances of atomic masses relative to iron enables to receive comparable results for different objects.

Xe isotopes $(\mathrm{A}=132,134,136)$ are stable products of $\beta$ - decay of fission fragments. Heavier decay products can serve as indicators of the contribution of fission fragments of actinide nuclides, and the lighter ones - of the contribution of superheavy nuclides fission.

The purpose of this report is to show the signatures of presence of fission fragments in matter of objects of the Solar system which have retained "memory" of a primary star nuclei synthesis of their heaviest progenitors.

\section{Cumulative atomic mass distributions in matter of the Solar system.}

The elemental abundances in matter of various objects of the Solar system [Ref. 2 - 5 in the title of Fig. 1] have been used for the calculation of atomic mass distributions in these objects. Isotope yields for various elements are taken from [2]. Though these yields for the same elements can be different in various objects, the difference does not considerably change the cumulative mass distributions as do the distinctions in abundances of chemical elements in various objects, which are as well caused by their nucleosynthesis . 
The curves of mass distributions for some regions of transiron elements have some features which can be described by yields of fission fragments [1]. Two types of mass distributions are indicative of the presence in primordial matter of the Solar system of two, at least, basic nucleosynthetic sources of fission fragments.

One of them is fixed in carbonaceous chondrites and photosphere of the Sun, having the low abundances of uranium. The objects mentioned above are characterized by Dy, Pt and Te enrichment $(\mathrm{A}=164,195$ and 130). The even-odd fluctuations in the mass distribution related to s-process are weakened in peaks at mass numbers 130 and 164, and almost completely disappear at $\mathrm{A}=195$ (Fig. 1A). The latter can be explained by the general tendency in a decrease of abundance of $\mathrm{s}$ - process nuclides with an increase in their mass number. These peaks are connected usually with the significant contribution of the nuclei formed in $r$ - process - fast capture of neutrons by iron nuclei [2]. However, the mentioned features can arise as well upon addition of products of spontaneous fission of superheavy nuclides $(Z \approx 114$ and 126$)$ to $\mathrm{s}-$ process nuclides [1]. In non-magmatic iron meteorites IAB, the nuclide abundance in $\mathrm{A}=195$ area for which data are available, appears the highest among objects of Solar system and, hence, the content of a superheavy progenitor with $\mathrm{Z} \approx 126$ could be the highest as well (Fig. 3 in Ref. [1])

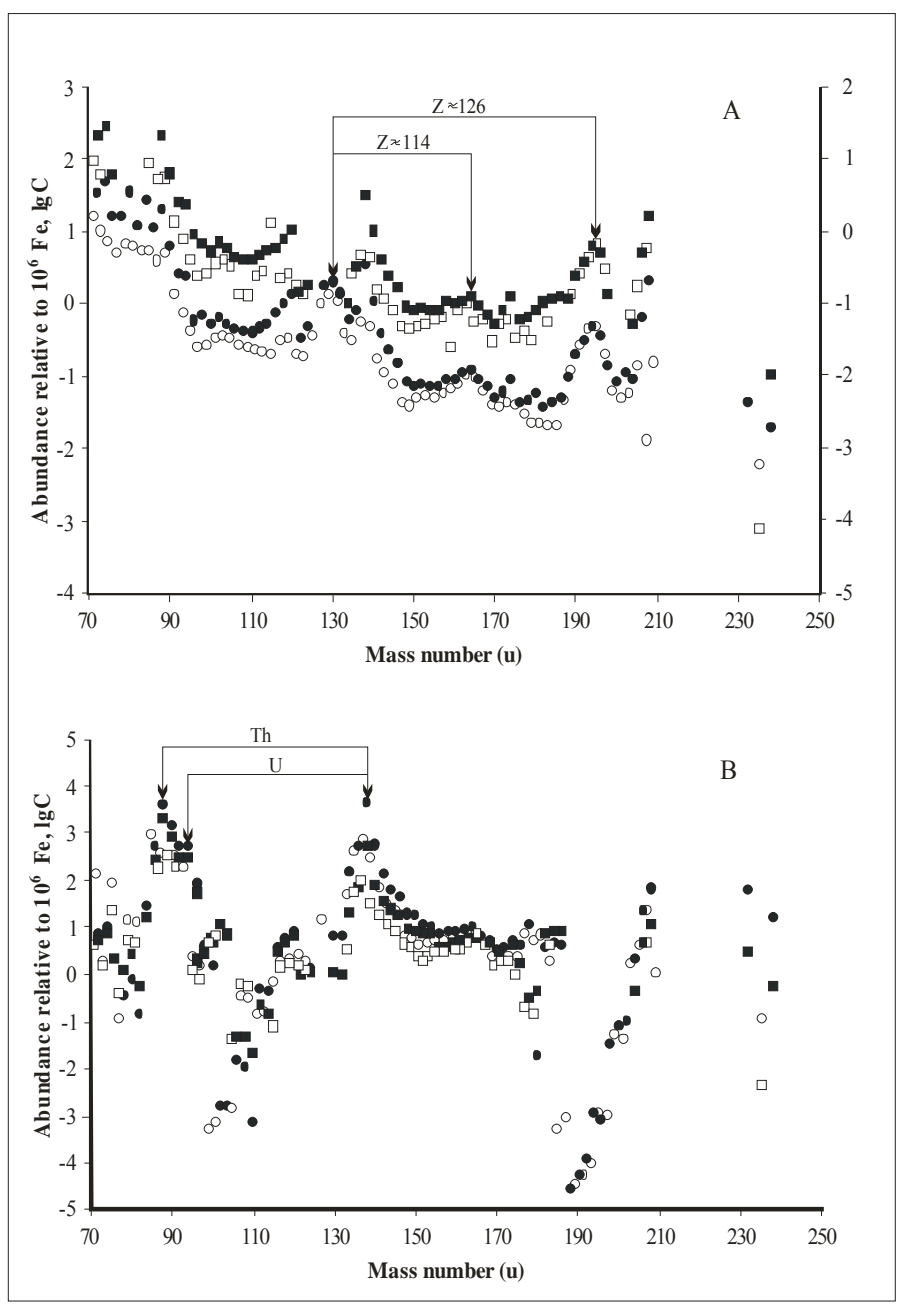

Figure 1: Atomic mass abundances in

A: carbonaceous chondrites

[2] (circles, abundances on the left axis) and the solar photosphere [3] (squares, abundances on the right axis);

B: upper continental crust of the Earth [4] (circles) and basalts of the Moon, "Luna 16" [5] (squares).

Closed and open symbols are even and odd atomic masses accordingly. Thin lines show the modes of mass distributions of light and heavy fragments in asymmetric fission of the possible heaviest nuclides. 
Other source of fission fragments can be seen in objects with high uranium contents: the Earth and the Moon crusts (Fig. 1B). There is a well expressed maximum A=138, and also some close located maxima of a different height $\mathrm{A}=88$ and 94 . A decrease of even-odd differences of the mass distribution is typical for them as well. Occurrence of these maxima is caused mainly by an enrichment of crusts of the Earth and the Moon in $\mathrm{Ba}, \mathrm{Sr}$ and $\mathrm{Zr}$, respectively. According to the rules of "unchanged charge density" and equality of $\beta$ - decay chains [6], the maxima near $A=88,94$ and 138 can correspond to light and heavy fragments of Th and U nuclear asymmetric fission. But the best coincidence with masses of the most longlived isotopes of these elements $\left({ }^{232} \mathrm{Th}\right.$ and $\left.{ }^{238} \mathrm{U}\right)$ turns out to be in the case of their fission in the excited state.

The estimated values of masses of natural parental nuclides and fragments of their fission before neutron emission are fairly well coordinated with the systematization of average masses for the prompt fission fragments after fission of actinide nuclides [6], $\mathrm{Rf}$ [7] and compound nuclei of superheavy elements in the vicinity or below the Coulomb barrier [8] (Fig. 2).

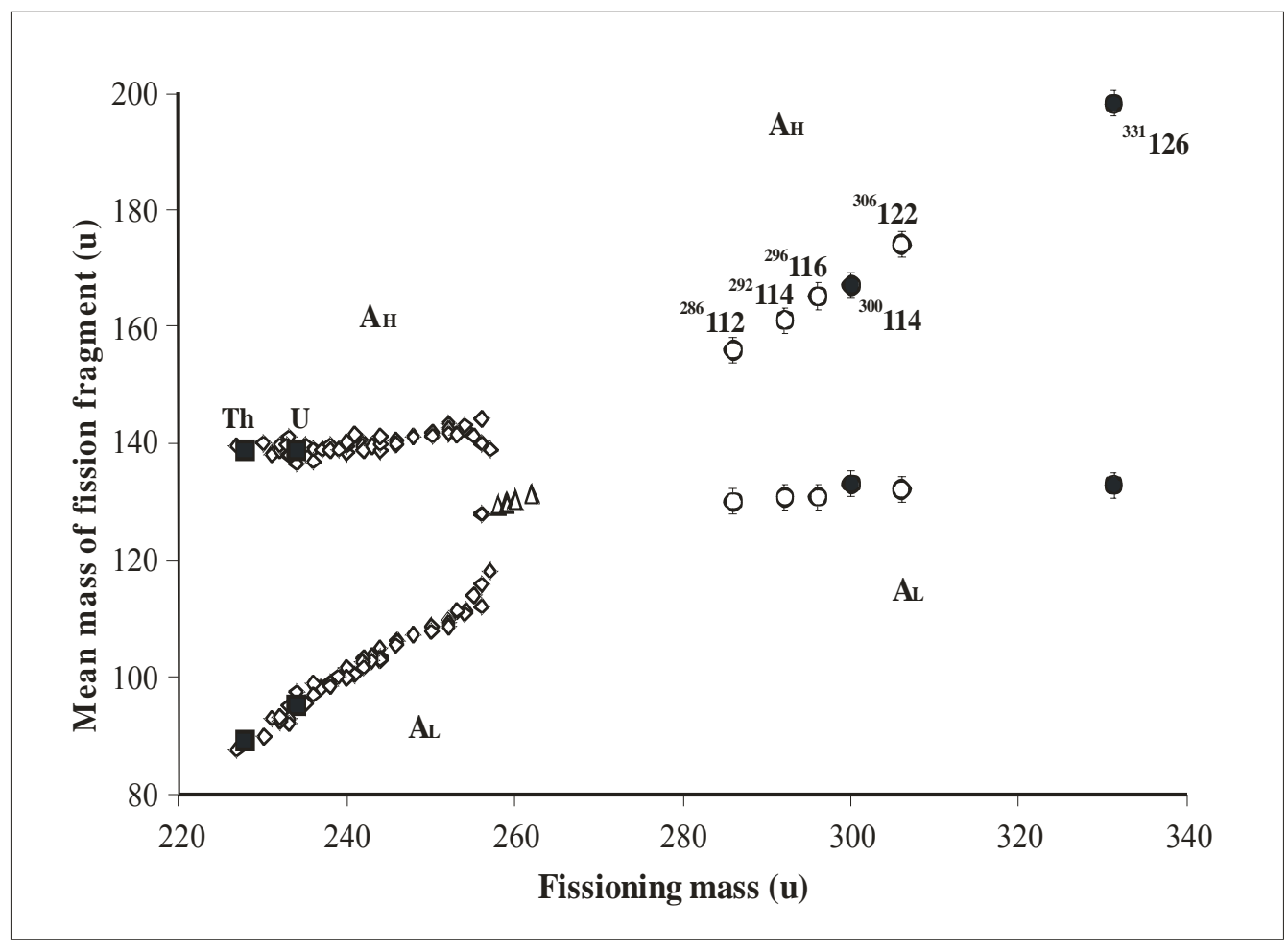

Figure 2: Positions of modes of mass distributions of fission fragments for the possible natural heaviest nuclides (closed circles and squares) on the plot of dependence of light (AL) and heavy (AH) fragment masses on the fissioning masses: of actinide nuclides (open diamonds) [6], of $\mathrm{Rf}$ isotopes (open triangles) [7], of compound nuclei in the superheavy region (open circles) [8]. Upper and lower series of the data are charges and masses of the compound [8] and possible natural [1] superheavy nuclides, accordingly.

The abundance ratios among nuclides of light groups of fission fragments $(\mathrm{A}=88$ and 94) are about the same as those of their parent nuclides - Th and U, accordingly, in the Earth and the Moon crusts. The strong minimum on the curves of mass distribution which makes it 
possible to explain a downturn of the contribution of fission fragments of nuclide $Z \approx 126$, is seen in the area $A \approx 195$ (Fig. 1B). Maximum $A=164$ observed in carbonaceous chondrites is also present in this mass distribution curve.

Considering that the heights of fission barriers for Th, $\mathrm{U}$ and superheavy elements are in a rather narrow range of values $(5.5-7.0 \mathrm{MeV}$, according to [9]) it is possible to assume that their ratios are equal to those of total masses of their fission fragments and remainders of the parent nuclides. An approximate estimate shows, that in a remainder after the induced fission at supernova explosions and in jet flows from neutron stars no more than $10^{-3}$ parent nuclei should be left. The calculated value allows the abundance of superheavy elements (SHE) to be estimated in various natural objects depending on their lifetimes.

\section{Contributions of fission fragments to xenon isotopes}

Numerous analyses of the ratios of heavy Xe isotopes $(A=132,134,136)$ testify that the mixture in substance of the Solar system is mainly a two-component one (Fig. 3). It could be formed as a result of fission fragments contribution input of the two basic sources of synthesis of parent nuclei for them.

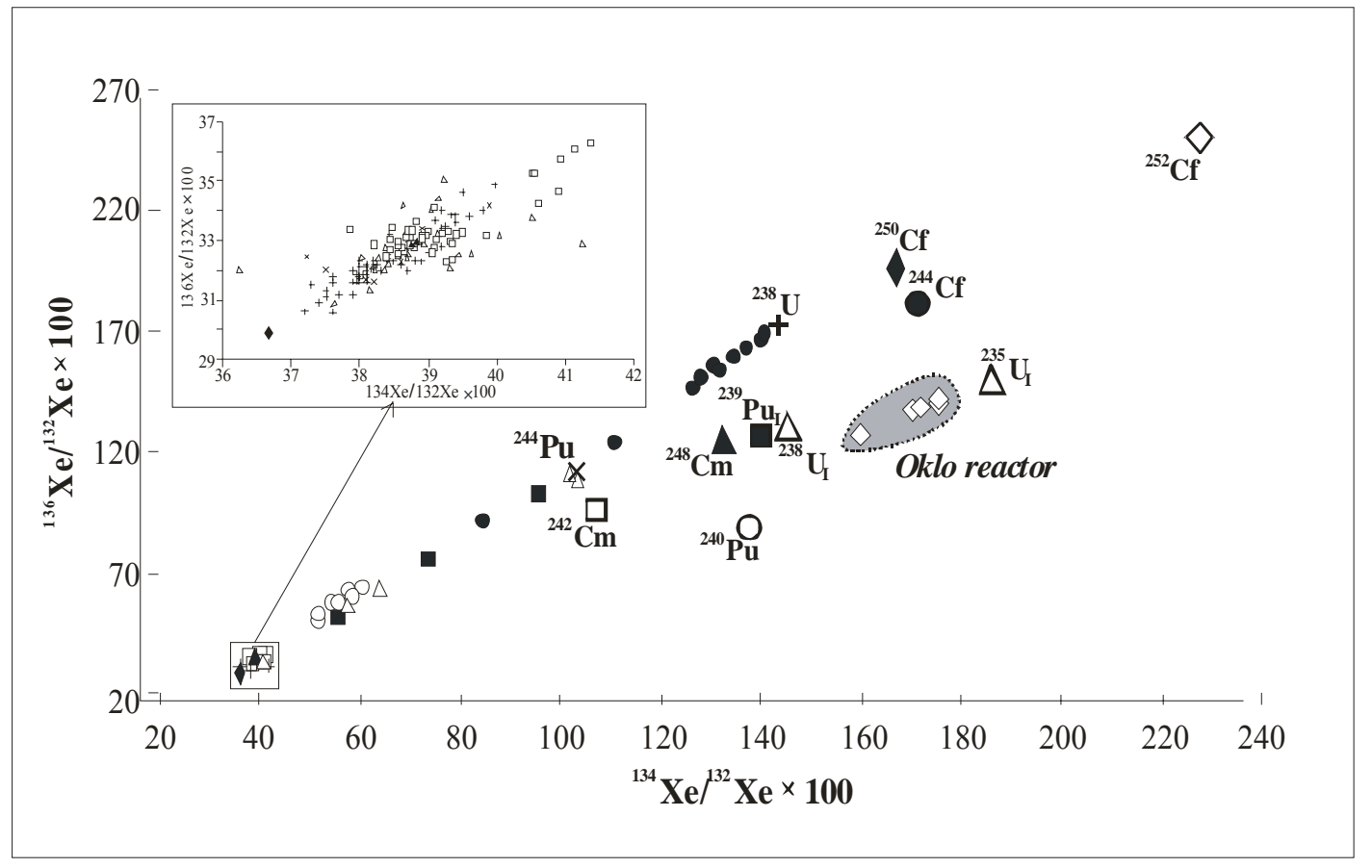

Figure 3: ${ }^{136} \mathrm{Xe} /{ }^{132} \mathrm{Xe}$ versus ${ }^{134} \mathrm{Xe} /{ }^{132} \mathrm{Xe}$ ratio for the xenon released from products of spontaneous and neutron-induced (I) fission of actinide nuclides and matter of different objects of the Solar system: carbonaceous chondrites (straight crosses) [10], ordinary chondrites (inclined crosses), iron meteorites (open triangles on the inset) [11], achondrites (closed squares) [11, 12], nanodiamonds (open cirles) [13], Earth's mantle gases (open squares) [14, 15, 16], Earth atmosphere (closed triangle) [15], Solar wind (close diamond) [14], ancient zircons (closed cirles) [17], Oklo natural nuclear reactor [18], Sudbury norite retained at various temperatures (open triangles) [19], products of spontaneous fission: ${ }^{244} \mathrm{Pu}$ and ${ }^{238} \mathrm{U}$ (Ref. 5 and 32 in [20]), ${ }^{240} \mathrm{Pu},{ }^{242} \mathrm{Cm},{ }^{244} \mathrm{Cm},{ }^{248} \mathrm{Cm},{ }^{250} \mathrm{Cf}$ and ${ }^{252} \mathrm{Cf}[11]$; products of neutroninduced fission: ${ }^{238} \mathrm{U}$ and ${ }^{235} \mathrm{U}$ (Ref. 38 in [20]), ${ }^{239} \mathrm{Pu}[21]$ 
An enrichment of substance in the lighter isotope ${ }^{132} \mathrm{Xe}$ can be a result of the greater contribution of fission fragments of superheavy nuclides. It is fixed in carbonaceous and ordinary chondrites, iron meteorites, the Solar wind as well as in deepness gases and in the atmosphere of the Earth. An enrichment in heavy xenon isotopes of nanodiamonds from meteorite Efremovka can be explained by addition of fission fragments from the second source of nuclei synthesis enriched with fission fragments of actinide nuclides.

The composition of xenon isotopes in the Earth's crust was formed under the influence of deepness degassing and the contribution of products of fragmental fission of uranium and ${ }^{244} \mathrm{Pu}$ after the Earth accretion. As a result of the hard accretion of the substance to be included into the Earth's crust, the substance loses Xe isotopes. While there are only very rare evidences of the presence of the given component in Xe isotope analyses in the samples of the Earth's crust impoverished by uranium (for example, Sudbury norite retained at various temperatures in Figure 3)

In Figure 3, in a limiting top position on a line of mixture there are ratios of xenon isotopes ${ }^{136} \mathrm{Xe} /{ }^{132} \mathrm{Xe}$ and ${ }^{134} \mathrm{Xe} /{ }^{132} \mathrm{Xe}$ in the products of spontaneous fission ${ }^{238} \mathrm{U}$. There are also some objects enriched with SHE fission products in a point with the minimal values of these ratios. At the same time ${ }^{136} \mathrm{Xe}$ and ${ }^{134} \mathrm{Xe}$ enrichment in achondrites, gases of the Earth's crust, ancient zircons (Fig. 3) is reached due to addition of products of spontaneous fission ${ }^{238} \mathrm{U}$ and ${ }^{244} \mathrm{Pu}$ after these objects were formed.

It is interesting to note, that the line of xenon isotope mixture is not in the field of points of neutron-induced fission of separate isotopes of actinide elements and samples from Oklo natural nuclear reactor where the neutron-induced fission also prevails. This means that the positions of humps on the curves of atomic mass distributions (Fig. 1) are analogous to those of on the distribution curves at spontaneous fission.

\section{Conclusions}

The explanation of a way and time of formation of actinide and superheavy nuclides during star nucleosynthesis can be based on the following facts from the mass distributions of chemical elements and xenon isotope ratios in substance of the Solar system. The masses of parental nuclides of thorium and uranium (228 and 234 accordingly), found out as the sums of fission fragments $(\mathrm{A}=88,94$ and 138) and approximately two neutrons emitted at spontaneous fission appear to be by 4 a.m.u. less than their real value for their long-lived isotopes ${ }^{232} \mathrm{Th}$ and ${ }^{238} \mathrm{U}$. This result can mean that fission of ${ }^{232} \mathrm{Th}$ and ${ }^{238} \mathrm{U}$ nuclei occurred from the excited state. It is well known that neutron multiplicity at fission increases by one upon excitation of a nucleus with every $7-8 \mathrm{MeV}$ [6]. It leads to the value of $30 \pm 2 \mathrm{MeV}$ for the excited state energy of these nuclei at the moment of the induced fission.

The specified excitation of nuclei is not connected with $r$ - process as the trajectory of ratios of heavy xenon isotopes in matter of the Solar system passes outside of a field of the neutroninduced fission of actinide nuclei (Fig. 3). It is possible to exclude also the processes of ${ }^{232} \mathrm{Th}$ and ${ }^{238} \mathrm{U}$ photofission that are responsible for the distributions of xenon isotopes which are similar to the distributions after fission by neutrons [22]. In this case, neutrino-induced fission 
can appear more realistic [23]; however, mass distributions of fission fragments caused by one are not known yet.

The small widths at half maxima of the mass distribution of fission fragments ( $\sim 8$ a.m.u.) in Figures $1 \mathrm{~A}$ and $1 \mathrm{~B}$ are not characteristic for nuclei fission. The very narrow hump observed for ${ }^{260} \mathrm{Md}$ fission distribution and explained by the shell effect [24], is a rare experimental fact in the vicinity of the area of superheavy elements. This phenomenon (Fig. 1) can be connected to the subsequent fast capture of neutrons by fission fragments in r-process. It should lead to a narrowing of humps of the mass distribution for the fragments, having a magic number of neutrons. Indeed, the maxima $A=88,94 ; 130$ and 138, on the one hand, as well as the maximum 195 , on the other hand, are under influence of the so-called closed neutron shells $\mathrm{N}=50,82$ and 126, accordingly. Atomic nuclei with these "magic" neutron numbers are fairly stable towards neutron capture. Narrow mass distributions in the "waiting points" of $r$ - process are well reproduced in the theoretical models wherein the possible contribution of fission in the final mass abundances has been taken into account $[25,26]$.

In the scenario suggested by the author the basic phase of $r$ - process proceeds after synthesis and fission of actinide and superheavy nuclides and this is the reason of local modifications in humps of their fission fragments distributions which coincide with the "waiting points" of $r$ - process. In author's opinion, with this scenario it is possible to explain also the origin of a controversial component "CCFXe" (carbonaceous chondrite fission xenon), enriched in the heavy $\mathrm{Xe}$ isotopes and concentrated mainly in the host phases of carbonaceous chondrites [27]. The most probable source of CCFXe can be neutrino-induced fission of actinide nuclei which bring the main contribution to this component. The subsequent $r$ - process could modify the distribution of fission fragments, leading to a significant enrichment of the heaviest Xe isotope $-{ }^{136} \mathrm{Xe}$. Absence of evidence for fission tracks in the host phases and into adjacent phases of carbonaceous chondrites indicates at the trapped origin of CCFXe. An enrichment in heavy Xe isotopes in achondrites, on other hand, is caused by ${ }^{244} \mathrm{Pu}$ spontaneous fission in situ that is proved also by a spectrum of distribution of heavy Xe isotopes and the presence of superfluous (over ${ }^{238} \mathrm{U}$ ) tracks of spontaneous fission.

Modeling of conditions of synthesis of actinide- and superheavy nuclides in view of the revealed facts is an object of research in the future. Synthesis of superheavy nuclides in $r$ process at supernova explosions and in jets of relativistic particles, collimated by a magnetic field of neutron stars, is the prevailing assumption in the recent publications [23, 28]. However, one can see that nucleosynthesis of actinide and superheavy elements could proceed before the basic neutron flow appears.

The formation of actinide- and superheavy nuclides before $r$ - process is supposed to take place in the core of massive presupernovae and crust of neutron stars where ${ }^{56} \mathrm{Fe}$ nuclei dominate. Consideration of interaction between ${ }^{56} \mathrm{Fe}$ nuclei, closely packed in clusters here [29], can give interesting results for the transition of nucleosynthesis into actinide and superheavy regions. The number of ${ }^{56} \mathrm{Fe}$ nuclei in these clusters should grow as pressure increases. Structures of the packing necessary for the synthesis of heaviest nuclides are as follows: tetrahedron cluster of ${ }^{56} \mathrm{Fe}$ nuclei $(\mathrm{A}=224)$ can give actinide nuclei; triangle dypyramid (five ${ }^{56} \mathrm{Fe}$ nuclei in dense packing cluster, $\left.\mathrm{A}=280\right)$ and octahedron $(\mathrm{A}=336)$ clusters can lead to the formation of superheavy nuclides $Z \approx 114$ and $Z \approx 126$, accordingly. Neutronization of 
iron nuclei can essentially lower the Coulomb barrier to fusion into the heaviest nuclei, most stable under the given conditions. The nucleosynthetic component, enriched with fission products of $\quad Z \approx 126$ (cosmic rays, iron meteorites, carbonaceous chondrites [1]) should differ in a higher compression of matter in a star to lead to polyhedron with the greatest number of tops - octahedron cluster.

\section{Acknowledgments}

Author is grateful to Professors Yu.Ts. Oganessian, M.G. Itkis and S.N. Dmitriev for stimulating discussions concerning the problems of superheavy elements. This work was supported in part by the Russian Foundation of Basic Research under Grant 04-02-17447.

\section{References}

[1] G.N. Goncharov, Nucleosynthesis and search for superheavy elements in nature: the possible scenario, Proc. Int. Symp. on Exotic Nuclei "EXON 2004", World Sc. Publ. Comp., Singapore, 279-284 (2005). http://flerovlab.jinr.ru/flnr/proceed.html

[2] E. Anders and N. Grevesse, Abundances of the elements: Meteoritic and solar. Geoch. Cosm. Acta. 53, 197 - 214 (1989).

[3] N. Grevesse and A.J. Sauval, The composition of the solar photosphere. Adv. Space Res. 30, 3-11 (2002).

[4] R.L. Rudnick and S. Gao, Composition of the continental crust. Treatise on geochemistry. 3.01, 1$64(2003)$

[5] A.P. Vinogradov, Preliminary data on lunar soil brought by Luna 16 automatic station. In "Lunar soil from Sea of Fertility". Izdatel'stvo Nauka. Moscow. 7 - 18 (1974) http://adsabs.harvard.edu/abs/1974lssf.book

[6] Yu.P. Gangrsky, B.N. Markov and V.P. Perelygin, Registration and spectrometry of fission fragments. Energoatomizdat. Moscow. (1992)

[7] E.K. Hulet, Spontaneous fission in the heavy elements. Proc. 3rd Int.Conf. Dynam. aspects of nucl. fission, Casta-Papiernicka, Slovak Rep. 1996. Dubna (JINR). 218 - 230 (1996)

[8] M.G. Itkis, et.al., Fusion - fission of heavy and superheavy nuclei. Phys. At. Nucl. 66, 1118-1124 (2003). http://www.springerlink.com/content/1562-692X/

[9] A. Mamdouh, J.M. Pearson, M Rayet and F. Tondeur, Fission barriers of neutron-rich and superheavy nuclei calculated with the ETFSI method. Nucl. Phys. A679, 337-358 (2001)

[10] E. Mazor, D. Heymann and E. Anders, Noble gases in carbonaceous chondrites. Geoch. Cosm. Acta. 34, $781-824$ (1970)

[11] Yu.A. Shukolyukov. and Dang Vu Minh, Fission products of transuranium elements in cosmos. Izdatel'stvo Nauka. Moscow. (1984)

[12] J.D. Gilmour, J.A. Whitby and G. Turner, Disentangling xenon components in Nakhla: Martian atmosphere, spallation and Martian interior. Geoch. Cosm. Acta. 65, 343- 354 (2001) 
[13] J.D. Gilmour, et. al., Xenon isotopes in size separated nanodiamonds from Efremovka: ${ }^{129} \mathrm{Xe}, \mathrm{Xe}-$ $P 3$, and Xe-P6. Geoch. Cosm. Acta. 69, $4133-4148$ (2005)

[14] M.W. Caffee, et.al., Primordial noble gases from Earth's mantle: identification of a primitive volatile component. Science. 285, 2115 - 2118 (1999)

[15] T.Matsumoto, et.al., Noble gases in pyroxenites and metasomatised peridotites from the Newer Volcanics, southeastern Australia: implications for mantle metasomatism. Chemical geology. 168, $49-73(2000)$

[16] R.Yokochi and B.Marty, Geochemical constraints on mantle dynamics in the Hadean. Earth Pl. Sc. Let. 238. 17- 30. (2005)

[17] G. Turner, et. al., Extinct ${ }^{244} \mathrm{Pu}$ in ancient zircons. Science. 306, 89 - 91 (2004)

[18] Yu.A. Shukolyukov et.al., Isotopic studies of the natural nuclear reactor. Geochem. Int. 14, No4, $12-27$ (1977)

[19] W.L. Raines, T.M.Flanders and P.K.Kuroda, Superheavy elements in nature?: Fissiogenic xenon in Sudbury, Ontario, norite. Geochemical Journal. 12, 265 - 270 (1978).

[20 ] M. Honda, A.P. Nutman and V.C. Bennett, Xenon compositions of magmatic zircons in 3.64 and 3.81 Ga meta-granitoids from Greenland - a search for extinct ${ }^{244} \mathrm{Pu}$ in ancient terrestrial rocks. Earth P1. Sc. Let. 207, 69 - 82 (2003)

[21] H. Ihara et. al., JNDS FR Decay and Yield Data. JAERI, Japan (1981)

[22] Yu.P.Gangrsky, et.al., Independent yields of Kr and Xe isotopes in the photofission of heavy nuclei. Phys. At. Nucl. 66, 1211-1218 (2003)

[23] Y.-Z. Qian, Neutrino-induced fission and r-process nucleosynthesis. Astroph. Journ. 569. L103 L106 (2002)

[24] E.K Hulet, et. al., Spontaneous fission properties of ${ }^{258} \mathrm{Fm},{ }^{259} \mathrm{Md},{ }^{260} \mathrm{Md},{ }^{258} \mathrm{No}$, and ${ }^{260}[104]$ : Bimodal fission. Phys. Rev. C. 40, 770 - 784 (1989)

[25] G. Martinez-Pinedo et. al., Nucleosynthesis in neutrino heated matter: The v $\rho$-process and the $r-$ process. Proc. Int. Symp. « NIC-IX», CERN, Geneva, June 25-30, 2006. PoS (NIC-IX) 064, (2006)

[26] I.V. Panov and I.Yu Korneev, Fission recycling in the $r$-process and formation of the second peak with $A \sim 130$. ibid., 156, (2006)

[27] E. Anders, Noble Gases in Meteorites: Evidence for Presolar Matter and Superheavy Elements. Proc. Roy. Soc. Lond.. A. 374, 207-238 (1981)

[28] A.G.W. Cameron, Some nucleosynthesis effects associated with $r$-process jets Astroph. Journ. 587. 327-340 (2003).

[29] J.M. Lattimer and M. Prakash, The physics of neutron stars. Science. 304, 536 - 542 (2004) 